\section{Effects of person-group and intragroup attitude similarity on perceived group attractiveness and cohesiveness}

\author{
LAWRENCE R. GOOD \\ Middle Tennessee State University, Murfreesboro, Tenn. 37130 \\ and \\ DON A. NELSON \\ Purdue University, Lafayette, Ind. 47907
}

The generalizability of the Byrne-Nelson attraction function to the evaluation of group attractiveness and group cohesiveness was investigated by varying both the proportion of person-group attitude similarity $(.25, .50$, or .75) and the proportion of intragroup similarity $(.33, .50$, or .83$)$ and having the experimental $S$ evaluate the stimulus group for its attractiveness and its probable level of cohesive functioning. Group attractiveness was measured by scales for liking and desire to work with the group, and group cohesiveness was assessed with scales asking for evaluations of the group's probable level of productivity, efficiency, feelings of belongingness, and morale. It was found that the evaluation of group attractiveness is a positive function of person-group attitude similarity $(p<.001)$, whereas the evaluation of group cohesiveness is a positive function of intragroup similarity or the similarity of the group members to one another, independent of their similarity to the nonmember evaluator $(p<.001)$.

Attitude similarity has been shown to affect interpersonal attraction in an attitude-attraction paradigm developed by Byrne (reviewed in Byrne, 1969). The procedure utilized in the typical attraction study involves having the experimental $S$ complete an opinion survey concerning a heterogeneous set of topics and, during a later session, evaluate a stranger on the basis of his purported responses to the same opinion survey, the stranger's responses being manipulated by the $E$ to present the $S$ with a predetermined level of similarity. The task is presented to the $S$ as an investigation of judgmental processes wherein he is to attempt to make accurate judgments about the stranger's intelligence, knowledge of current events, adjustment, and morality on the basis of knowing some of his attitudes. In addition, the $\mathrm{S}$ is asked to estimate how much he would like the stranger and how much he would desire him as a work partner in an experiment, these last two 7-point scales of the Interpersonal Judgment Scale (IJS) typically being summed to constitute a 2- to 14-point attraction measure for which a split-half reliability of .85 has been obtained (Byrne \& Nelson, 1965).

It has been demonstrated that $\mathrm{Ss}$ respond to the proportion of similar attitudes rather than to the number of either similar or dissimilar attitudes and that the functional nature of the similarity-attraction relationship is essentially a linear one suggesting an empirical S-R law of attraction (Byrne \& Nelson, 1965). The generalizability of the Byrne-Nelson function has been with himself as determined from inferences the $E$ pretends to have drawn from a trait questionnaire the $S$ had completed prior to the experiment (e.g., Back, 1951). This commonly employed stimulus manipulation confounds at least two aspects of the stimulus variable, however, since it is unclear whether the group's subsequent "cohesiveness" is a function of telling the $S$ that his partner and he "should get along extremely well [Back, p. 12]" or, rather, a function of the E's vague intimations to the $S$ about a successful "match" derived from the trait-questionnaire material. In cohesiveness research where attraction has been examined as a dependent variable and operationalized via sociometric-choice indices (e.g., Schachter, 1951), on the other hand, the resulting data are more clearly interpretable as delineating attraction to the individual rather than attraction to the group, and the effects between-group differences in average opinion similarity might have on attraction to the group as a whole cannot be evaluated due to the limitation imposed by the sociometric methodology.

The present study was therefore designed to evaluate how the actual manipulation of attitude similarity-dissimilarity would affect ratings of attraction to the group, it being hypothesized that a group's attractiveness, as perceived by one who was not a member of the group, is a positive function of the degree of attitudinal similarity he shares with the group members. Since within-group or intragroup similarity can vary independently of the nonmember's own similarity to the group, it was felt that this element of the stimulus should be controlled and systematically varied as a separate factor in a two-way experimental design. Furthermore, it was felt that a group's perceived attractiveness and its perceived cohesiveness should be evaluated as separate response variables, since it seemed reasonable to suppose that a group could be perceived as being highly cohesive by the outside observer but not as having members attractive to himself. The degree of intragroup attitude similarity, then, would presumably be the factor determining the nonmember's evaluation of a group's cohesiveness, irrespective of the nonmember's own similarity to the group. It was therefore hypothesized that perceived group cohesiveness is a positive function of intragroup attitude similarity.

\section{METHOD}

Introductory psychology students were asked to complete a 12-item 
survey of attitudes concerning such topics as undergrduates getting married, admission of Red China to the U.N., and bussing to attain racial integration. Each attitude was presented as a 6-point scale for varying degrees of agreement and disagreement. To preserve the anonymity of the S's responses, he was asked to indicate his mother's maiden name instead of his own name, and this name was then used as his code name in the study. During a class period 9 to 14 days later, the $S$ received a packet including an instruction sheet and three attitude surveys which had purportedly been filled out by three individuals who were to comprise a proposed three-person discussion group. To enhance the credibility of these stimulus persons, the $\mathrm{E}$ had cut out the portion of each group member's attitude survey calling for the respondent's name and other personal information, used different types of pencils and pencil marks when manipulating the attitudes of the members of each stimulus group, and employed the constant-discrepancy technique (Byrne, 1969) for the manipulation of attitude similarity-dissimilarity. The instruction sheet stated that the formation of several small discussion groups comprising interested student volunteers was being considered for another psychological experiment and that the present concern was determining the extent to which individuals who were not members of these groups could form valid judgments about the groups (see Good, 1971, for the verbatim version of the instructions). After the Ss had had the opportunity to examine the three attitude surveys they had received, the group judgment scale, representing a modified form of the Byrne IJS, was passed out; this consisted of six 7-point scales calling for evaluations of probable level of productivity, efficiency, feelings of belongingness, morale, how much the $S$ would probably like the group, and how much he would enjoy working with the group in an experiment.

The mythical groups which the Ss evaluated comprised three bogus

\begin{tabular}{|c|c|c|c|}
\hline \multirow{2}{*}{$\begin{array}{l}\text { Attitude } \\
\text { Item }\end{array}$} & \multicolumn{2}{|c|}{ Group } & Nember \\
\hline & 1 & 2 & 3 \\
\hline 1 & + & - & + \\
\hline 2 & + & + & - \\
\hline 3 & - & + & + \\
\hline 4 & - & - & - \\
\hline 5 & + & - & + \\
\hline 6 & + & + & - \\
\hline 7 & - & + & + \\
\hline 8 & - & - & - \\
\hline 9 & + & - & + \\
\hline 10 & + & + & - \\
\hline 11 & - & + & + \\
\hline 12 & - & - & - \\
\hline
\end{tabular}

strangers, all with attitudes $25 \%, 50 \%$, or $75 \%$ similar to those of the S. In addition, the within-group or intragroup similarity was varied by making the three group members $33 \%$, $50 \%$, or $83 \%$ similar to each other. This last experimental manipulation was made possible by systematically varying the sequence of similarity-dissimilarity across the 12-item attitude survey for the members of each triad, as illustrated in Table 1 , for the $50 \%$ person-group-similarity $50 \%$ intragroup-similarity condition, where a "plus" indicates an attitude agreeing with the S's and a "minus" an attitude disagreeing with the S's. It was not possible, however, to construct a full nine-celled 3 by 3 (Intragroup by Person-Group) factorial design in that $33 \%$ intragroup similarity could be attained only at the $50 \%$ person-group-similarity level. Hence a 2 by 3 design was employed with an additional cell for $33 \%$ intragroup similarity at the $50 \%$ person-group-similarity level in anticipation that a separate single-factor analysis of variance could be computed across this level of person-group similarity. Ss were assigned randomly to the treatment conditions, and, to facilitate the utilization of equal-cell statistical procedures, the group judgment scales of 21 Ss in the original S pool were randomly discarded, leaving 24 Ss (13 male and 11 female) in each of the seven treatment cells for a total final sample of $168 \mathrm{Ss}$.

The first four scales (productivity, efficiency, feelings of belongingness, and morale) of the group judgment scale were conceptualized as forming a four-item 4 - to 28-point measure of perceived group cohesiveness, and a check on the reliability of this measure was made using the Spearman-Brown formula. The odd-even correlation for the four scales was found to be .64 , yielding a split-half reliability estimate of $.77(\mathrm{~N}=168)$. The last two scales of the group judgment scale (liking and desire to work with the group) were presumed to assess group attractiveness, and they were found to correlate .71 with each other, yielding a split-half reliability of .82 for the combined 2-to 14-point measure.

\section{RESULTS}

The means and standard deviations for the evaluations of group attractiveness are shown in Table 2. The evaluations of attractiveness increased with the level of attitudinal similarity shared by the $S$ and the group members, a person-group similarity main effect which was highly significant $(F=14.19$, $\mathrm{df}=2 / 138, p<.001$ ). The overall linear trend was also highly significant $(F=28.12, \quad d f=1 / 138, \quad p<.001)$, while the quadratic was not $(F<1$, $\mathrm{df}=1 / 138)$. The group-attractiveness evaluations, however, were not significantly affected by the intragroup-similarity factor either for the 2 by 3 analysis of variance $(F=1.69, \mathrm{df}=1 / 138)$ or the separate single-factor analysis of variance com puted at the $50 \%$ person-group-similarity level to include the $33 \%$ intragroup-similarity cell $(\mathrm{F}<1, \mathrm{df}=2 / 69)$. The $\mathrm{AB}$ interaction was also nonsignificant $(F<1$, $\mathrm{df}=2 / 138$ ). These results support the hypothesis that the degree of attitude similarity one shares with a Group's members is a determinant of his attraction to the group in a fashion consistent with findings obtained in a single-stranger attitude-attraction research.

The means and standard deviations for the evaluations of group cohesiveness are given in Table 3 , and it is apparent that the ratings of cohesiveness varied as a function of intragroup rather than person-group similarity. Whereas the effect of the person-group-similarity factor was a
Table 2

Means and Standard Deviations of Evaluations of Group Attractiveness

\begin{tabular}{|c|c|c|c|c|c|c|}
\hline \multirow{3}{*}{$\begin{array}{l}\text { Propor- } \\
\text { tion of } \\
\text { Intra- } \\
\text { group } \\
\text { Similarity }\end{array}$} & \multicolumn{4}{|c|}{ Proportion of Person-Gro } & \multicolumn{2}{|l|}{ Similarity } \\
\hline & \multicolumn{2}{|c|}{.25} & \multicolumn{2}{|c|}{.50} & \multicolumn{2}{|c|}{.75} \\
\hline & Mean & SD & Mean & SD & Mean & $\mathrm{SD}$ \\
\hline .83 & 7.46 & 2.69 & 9.17 & 2.70 & 10.04 & 2.24 \\
\hline .50 & 6.92 & 2.62 & 8.38 & 2.55 & 9.75 & 2.17 \\
\hline .33 & - & - & 8.46 & 3.22 & - & - \\
\hline
\end{tabular}

Table 3

Means and Standard Deviations of Evaluations of Group Cohesiveness

\begin{tabular}{|c|c|c|c|c|c|c|}
\hline \multirow{3}{*}{$\begin{array}{l}\text { Propor- } \\
\text { tion of } \\
\text { Intra- } \\
\text { group } \\
\text { Similarity }\end{array}$} & \multicolumn{4}{|c|}{ Proportion of Person-Group } & \multicolumn{2}{|l|}{ Similarity } \\
\hline & \multicolumn{2}{|c|}{.25} & \multicolumn{2}{|c|}{.50} & \multicolumn{2}{|c|}{.75} \\
\hline & Mean & $\mathrm{SD}$ & Wean & SD & Mean & SD \\
\hline .83 & 19.71 & 2.58 & 20.38 & 2.62 & 20.83 & 3.55 \\
\hline .50 & 16.08 & 4.13 & 17.08 & 2.59 & 17.38 & 3.74 \\
\hline .33 & - & - & 15.75 & 3.90 & - & - \\
\hline
\end{tabular}


nonsignificant one $(F=1.73$, $\mathrm{df}=2 / 138$ ), the intragroup-similarity factor yielded a highly significant effect $(F=40.50, \quad d f=1 / 138$, $p<.001$ ) for the 2 by 3 analysis of variance and also for the separate single-factor analysis of variance carried out at the $50 \%$ person-group-similarity level to include the $33 \%$ intragroup cell $(F=14.18$, $\mathrm{d} f=2 / 69, \mathrm{p}<.001)$. The $\mathrm{AB}$ interaction was again nonsignificant ( $F<1, d f=2 / 138$ ). The hypothesis that intragroup attitude similarity functions as a determinant of the outsider's perception of a group's cohesiveness is thus clearly supported as greater cohesiveness was attributed to the more internally similar groups.

These analysis of variance results were essentially corroborated by analysis of covariance findings. Employing perceived cohesiveness as a covariate and perceived attractiveness as the dependent measure, the $F$ ratio for the intragroup-similarity factor was still nonsignificant $(F=1.52$, $\mathrm{df}=1 / 137)$. Using attractiveness as a covariate and cohesiveness as the dependent measure, the $F$ ratio for the person-group-similarity factor was also still nonsignificant $(F=.08$, $\mathrm{df}=2 / 137$ ).

\section{DISCUSSION}

The findings of the present study are interpreted as extending the generality of the Byrne-Nelson attraction function to the person-group context, with the nonmember S's attraction to a group as an entity being shown to be a positive function of the degree of attitudinal similarity he shares with the group's members. The distinction made between a group's attractiveness and its perceived cohesiveness as separate response variables was also found to be a meaningful one in the situation where a nonmember $S$ evaluates a stimulus group, since cohesiveness, as measured by ratings of productivity, efficiency, etc., was shown to be a positive function of the group's internal or intermember similarity rather than a function of S-group similarity. This latter effect suggests that individuals can appraise the probable outcomes of future interactions among persons comprising a group independently of their own personal feelings of attraction or repulsion toward the group, based upon an ability to respond differentially to the person-group and intragroup aspects of the attitudinal information they receive concerning the members of a group. This result, moreover, provides negative evidence for the proposition found in the cohesiveness literature (e.g., Festinger et al, 1950) to the effect that highly "cohesive" groups should be more attractive to outsiders than minimally cohesive groups, since ratings of attractiveness were not found to be dependent upon perceived cohesiveness in the present study.

It should be noted that the present results do not fully answer the question of whether Ss respond to the average proportion of attitudinal similarity across group members or to the additive sum of either similar or dissimilar attitudes held by the group members. A definitive answer to this question would presumably require the factorial manipulation of number as well as proportion of similar attitudes in stimulus groups of the sort carried out by Byrne \& Nelson (1965) for the single-stranger situation. On the basis of the present results, it is tentatively proposed that the nonmember's attraction to a group is a positive linear function of the average proportion of shared attitudinal similarity to the group and that the nonmember's perception of a group's cohesiveness is a positive linear function of the average proportion of intragroup attitude similarity. Although the present investigation was somewhat limited in scope, it is nonetheless hoped that the results may help to provide some conceptual and empirical linkage between the group cohesiveness and interpersonal-attraction research areas.

\section{BACK $K$ REFERENCES}

Influence through sociai In thencel of Abnormal \& Social Psychology, 1951, 46, 9-23.

BYRNE, D. Attitudes and attraction. In L. Berkowitz (Ed.), Adrances in experimental social psychology. Vol. 4. New York: Academic Press, 1969. Pp. 35-89.

BYRNE, D., \& NELSON, D. Attraction as a linear function of proportion of positive reinforcements. Journal of Personality \& Social Psy chology, 1965, 1, 659-663.

FESTINGER, L.. SCHACHTER, S.. \& BACK, K. Social pressures in informal groups. New York: Harper. 1950.

GOOD, L. R. Effects of intergroup and intragroup attitude simjlarity on perceived group attractiveness and cohesiveness. Unpublished doctoral dissertation, Purdue University, 1971.

SCHACHTER, S. Deviation, rejection, and communication. Joumal of Abnormal \& Social Psychology, 1951, 46, 190-207. 